UVX 2010 (2011) 131-137

DOI: $10.1051 / \mathrm{uvx} / 2011018$

(C) Owned by the authors, published by EDP Sciences, 2011

\title{
Diagnostic de sprays diesel par radiographie $X$
}

\author{
E. Robert, S. Dozias, R. Viladrosa, C. Cachoncinlle et J.-M. Pouvesle \\ GREMI, CNRS-Polytech'Orléans, 14 rue d'Issoudun, BP. 6744, 45067 Orléans Cedex 2, \\ France
}

\begin{abstract}
Résumé. Le travail présente la mise en œuvre d'un diagnostic à base de rayons $\mathrm{X}$ vers $8 \mathrm{keV}$ pour la caractérisation spatiale et temporelle dans la zone proche orifice, c'est-à-dire la région la plus dense, de sprays de diesel issus d'injecteur de série. Les principales propriétés de la source flash X sont décrites, domaine d'énergie, résolution spatiale, et géométrie de l'expérience de radiographie. Le principe et la précision de la mesure de densité des jets de carburant sont validés sur des objets tests. Les mesures traduisent l'existence de deux zones de densités et vitesse de propagation différentes sur les premières centaines de micromètre en aval de l'injecteur ainsi qu'un mélange relativement important du carburant liquide avec l'atmosphère gazeuse dans la chambre d'injection.
\end{abstract}

\section{INTRODUCTION}

L'optimisation des moteurs diesel est en constante évolution pour tenter d'aboutir à des régimes de combustion les plus efficaces possibles et pour limiter les émissions de suies, de dioxyde de carbone, d'hydrocarbures imbrûlés et autres sous produits nocifs pour l'environnement. En dehors de la mise au point récente de nouveaux carburants, «biodiesels», les moteurs diesel conventionnels fonctionnent avec des pressions d'injection de plus en plus élevées qui sont favorables pour l'atomisation du carburant et sa combustion. Cette évolution progressive va de paire avec un grand nombre de mesures expérimentales et de travaux de simulation pour la conception et la caractérisation de nouvelles stratégies d'injection (géométrie des injecteurs, durée et fréquence d'injection) et de chambre de combustion optimisées. Les techniques de mesures basées sur l'utilisation de faisceaux ou nappes laser ont ainsi permis la caractérisation des écoulements tant au niveau de la densité et de la taille des gouttelettes que leur distribution spatiale et leur dynamique. Ces techniques de référence pour les phases dispersées, gazeuses ou diphasique incluant des microgouttelettes, se heurtent néanmoins à des limitations physiques inhérentes à la propagation des faisceaux dans la zone la plus dense du spray en sortie d'injecteur. Face à l'inexistence de techniques de diagnostic fiables pour la caractérisation de l'écoulement sur des distances d'une longueur correspondant à quelques diamètres de l'orifice de l'injecteur, le développement des dispositifs a longtemps reposé sur les résultats issus des travaux de modélisation. Depuis quelques années, l'utilisation du rayonnement $\mathrm{X}$, sur la gamme d'énergie appropriée autour de $10 \mathrm{keV}$, a permis d'obtenir les premières cartographies, mesures de densité et de vitesse de propagation des sprays de carburant [1-4]. Ces premières mesures effectuées en exploitant la mono chromaticité et la grande résolution spatiale offertes par l'utilisation du rayonnement synchrotron ont permis de vérifier toute l'importance de recueillir des données de base pour les travaux de simulations. Cependant, le grand nombre de paramètres susceptibles d'influencer l'atomisation des sprays de carburant requiert de nombreuses études et motive le développement d'un diagnostic X plus flexible et d'accès plus facile basé sur l'emploi d'une source alternative au synchrotron.

Ce travail présente les résultats obtenus au laboratoire GREMI sur le diagnostic de sprays diesel issus d'injecteurs de série dans le cadre du programme Emphase supporté par le GIE PSA-Renault.

This is an Open Access article distributed under the terms of the Creative Commons Attribution-Noncommercial License 3.0, which permits unrestricted use, distribution, and reproduction in any noncommercial medium, provided the original work is properly cited. 


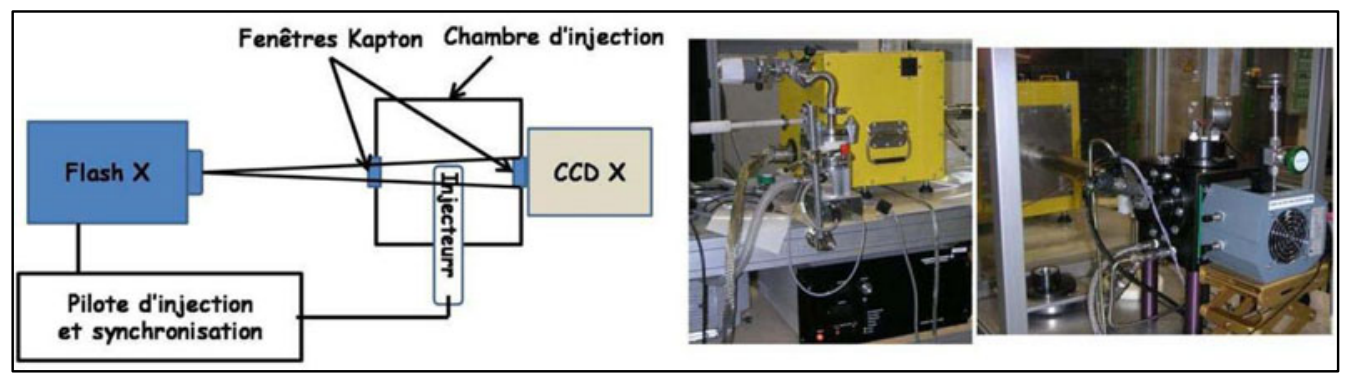

Figure 1. Dispositif expérimental. Sur la photographie du centre on observe le flash X, celle de droite présente la chambre d'injection (noire) et la CCD X.

L'objectif consistait à montrer la faisabilité de la visualisation et la caractérisation de la zone proche de la sortie des orifices micrométriques des injecteurs par radiographie $\mathrm{X}$ de basse énergie. Nous décrivons le dispositif expérimental et la mise en œuvre d'une source flash $\mathrm{X}$ adaptée, tant au niveau de l'énergie moyenne que de la dimension du foyer émetteur qui conditionne la résolution spatiale du diagnostic dans le paragraphe suivant. Le troisième paragraphe présente les radiographies de sprays d'hydrocarbure, et les mesures de la dynamique d'expansion, de l'angle d'ouverture et de la densité qu'elles ont permises sur des injecteurs de série ayant des orifices d'injection d'environ cent micromètres. Une synthèse et des perspectives de nos travaux sont finalement proposées dans la dernière section du manuscrit.

\section{DIAGNOTIC PAR FLASH $X$}

\subsection{Dispositif expérimental}

La figure 1 présente le dispositif expérimental. Une source de type «flash X» a été développée spécifiquement pour la caractérisation des sprays de carburant. Ce flash X est basé sur le principe de ceux mis en œuvre par l'équipe depuis plusieurs années [5] et émet des impulsions, d'une largeur temporelle typique de 20 nanosecondes, de rayons $\mathrm{X}$ dans la gamme d'énergie comprise entre 5 et $30 \mathrm{keV}$. Une optimisation spécifique et une caractérisation détaillée ont porté sur le spectre d'émission de la source et sur le meilleur compromis entre les dimensions du foyer émetteur et la stabilité des performances sur plusieurs dizaines de milliers de tirs [5,6]. L'injecteur est positionné à $75 \mathrm{~cm}$ du foyer $\mathrm{X}$ au sein d'une chambre d'injection, dont la pression de remplissage peut être contrôlée jusqu'à 30 bars, et qui est équipée de fenêtres minces $(125 \mu \mathrm{m})$ en Kapton ${ }^{\circledR}$ permettant une transmission importante du rayonnement X. Les injecteurs utilisés sont des injecteurs Bosch diesel de série ayant 5 ou 6 orifices d'un diamètre de 110 ou $150 \mu \mathrm{m}$. Une pièce spécifique, de type écorceur, est fixée au bout de l'injecteur. Cet écorceur conserve l'injection au travers de l'ensemble des orifices de l'injecteur mais permet de laisser propager dans la chambre d'injection uniquement un spray au travers d'un orifice orienté dans la direction verticale. Le fluide utilisé est du dodécane $\left(\mathrm{C}_{12} \mathrm{H}_{26}\right)$ dont les propriétés de viscosité et de densité sont très proches de celle des carburants diesel. L'enregistrement des radiographies est effectué à l'aide d'une caméra CCD X Andor DY432-FI-DD munie d'une fenêtre d'entrée en béryllium. Le pilote d'injection qui permet de fixer la pression du rail d'injection jusqu'à 1200 bars ainsi que la durée d'injection, de quelques centaines de microsecondes, est synchronisé avec les tirs de la source flash X. Compte tenu de la très faible absorption des rayons $\mathrm{X}$, de l'ordre de quelques pour cent, par les jets de carburant d'une épaisseur d'une centaine de micromètres, l'enregistrement des radiographies est effectué en moyennant les acquisitions sur quelques centaines à quelques milliers de cycles d'injection. 


\subsection{Performances du diagnostic par flash $X$}

La transmission des photons X vers la CCD s'effectue au travers de la chambre d'injection c'est à dire après la traversée d'une couche d'azote, à une pression jusqu'à 30 bars, d'une épaisseur de $10 \mathrm{~cm}$. Ceci impose la limite inférieure en énergie autour de $7 \mathrm{keV}$ pour garantir une transmission supérieure à $10 \%$ du flux incident. Par ailleurs, le calcul du rapport de transmission entre les photons X traversant le jet de carburant, d'une épaisseur de 110 à $150 \mu \mathrm{m}$, et la couche d'azote et ceux se propageant uniquement au travers de cette dernière, indique que le contraste devient inférieur à un pour cent pour des énergies supérieures à $10 \mathrm{keV}$. Ceci a conduit à développer une source utilisant une anode en cuivre pour optimiser la production de photons $\mathrm{X}$ dans la gamme d'énergie pertinente, ici autour de $8 \mathrm{keV}$. Un des objectifs des mesures étant l'estimation de la densité dans la zone proche orifice, il est nécessaire de pouvoir caractériser au mieux le spectre en énergie émis par le flash X. Le calcul de la densité repose sur l'inversion de la loi de Beer Lambert (1):

$$
\phi t(E)=\phi 0(E) \times \exp (-\sigma(E) N l)
$$

où $\phi_{t}$ et $\phi_{0}$ représentent les flux transmis et incident au travers d'une couche d'épaisseur 1 et de densité N. La transmission dépend de la section efficace $\sigma$ qui est une fonction de l'énergie $\mathrm{E}$ des photons X. Les couches d'azote en avant et en arrière du jet suivant l'axe de projection des rayons $\mathrm{X}$, se comportent comme des filtres atténuateur tant pour les photons traversant le spray que pour ceux se propageant en dehors du jet. Cette traversée de couches d'azote ne modifie pas le contraste radiographique intrinsèque entre le spray d'hydrocarbure et une couche de même épaisseur d'azote mais conduit à une très forte atténuation des flux reçus par la CCD. La radiographie fournit la mesure du rapport des flux transmis au travers du spray et incident sur la CDD, mesuré en dehors de l'axe du jet. Le calcul de la densité n'est rigoureusement possible que dans le cas où la section efficace est fixée c'est-à-dire dans le cas d'une source monochromatique. Cependant, dans le cas de source X présentant un spectre étendu, il est possible d'effectuer le calcul en estimant l'énergie moyenne équivalente du spectre réel [7]. Pour ceci, la radiographie d'objets tests de densité et épaisseur connues permet d'estimer l'énergie moyenne équivalente qui correspond le mieux à l'intégration sur l'ensemble du spectre émis par la source. Des mesures sur des films minces de Kapton®, de polymethylpentene (PTX®) et au travers de couches liquides d'eau ou de dodécane d'une épaisseur de $200 \mu \mathrm{m}$ maintenue entre deux films plastiques, indiquent une énergie moyenne de $8,3 \mathrm{keV}$. Cette valeur résulte de la contribution des raies du cuivre à $8 \mathrm{keV}$ et du rayonnement de freinage entre 5 et $30 \mathrm{keV}$. On déduit qu'une très grande majorité de l'énergie est émise dans les raies caractéristiques du matériau de l'anode en accord avec des mesures spectroscopiques [5,6]. Une fois l'énergie équivalente estimée, il s'agit de connaître la précision du diagnostic pour le calcul de la densité dans nos conditions expérimentales. Pour ceci, nous avons radiographié des fils de nylon d'un diamètre de 60, 100, 140 et $180 \mu \mathrm{m}$ et déduit l'écart relatif de la densité expérimentale avec celle de ces objets tests. La table 1 présente la précision de notre mesure pour les différents diamètres.

Pour des diamètres supérieurs à $100 \mu \mathrm{m}$, l'écart à la densité est inférieur à $15 \%$. Pour des diamètres inférieurs, la résolution spatiale du diagnostic dans les conditions géométriques de radiographie de sprays de diesel limite la précision de la mesure. En effet, la mesure, à l'aide de mires constituées de grilles en plomb à pas variable (motifs de type paires de lignes par mm), du contraste radiographique en fonction de la fréquence spatiale des motifs, conduit à une résolution spatiale de $120 \mu \mathrm{m}$ dans les

Tableau 1. Ecart des densités mesurées et réelles en fonction du diamètre d'un fil nylon. Une énergie équivalente de $8,3 \mathrm{keV}$ est prise en compte pour la mesure.

\begin{tabular}{|l|c|c|c|c|}
\hline Diamètre du fil nylon & $60 \mu \mathrm{m}$ & $100 \mu \mathrm{m}$ & $140 \mu \mathrm{m}$ & $180 \mu \mathrm{m}$ \\
\hline Ecart à la densité & $32 \%$ & $25 \%$ & $12 \%$ & $4 \%$ \\
\hline Incertitude & $13 \%$ & $13 \%$ & $11 \%$ & $2 \%$ \\
\hline
\end{tabular}




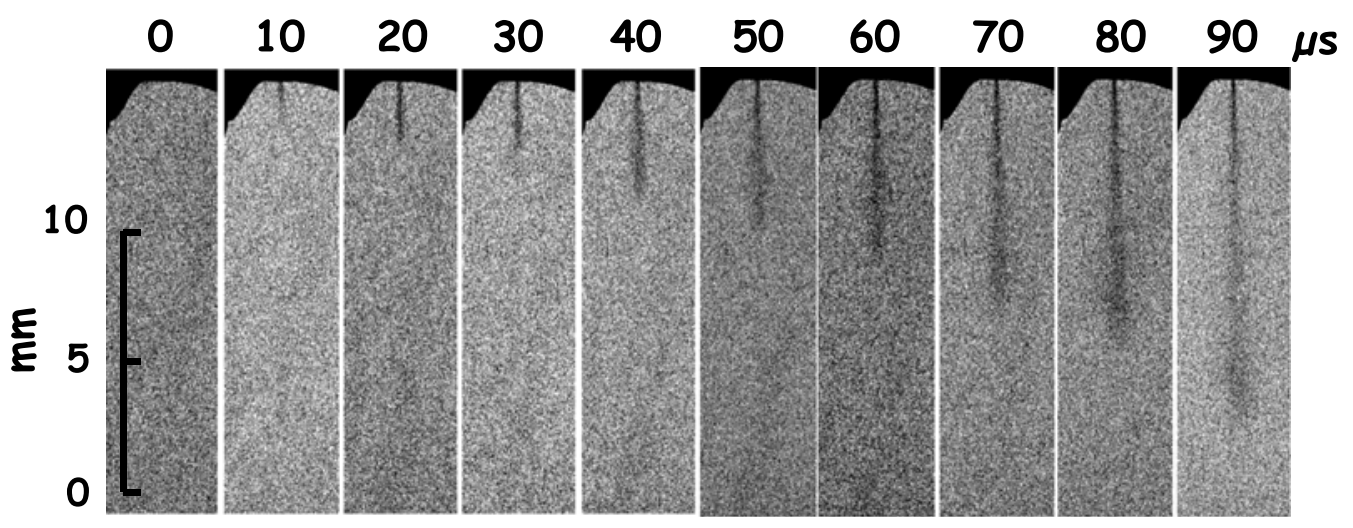

Figure 2. Radiographies du spray au travers d'un injecteur à 6 trous d'un diamètre de $110 \mu \mathrm{m}$. La pression de rail est de 800 bars, la durée d'injection de $500 \mu \mathrm{s}$, la chambre d'injection est à la pression atmosphérique. Chaque cliché est le résultat d'une moyenne sur 1000 tirs. L'incrément en temps est de $10 \mu$ s du début de l'injection (à gauche) jusqu'à $90 \mu$ s après sur la radiographie de droite pour laquelle l'expansion est mesurée sur $14 \mathrm{~mm}$.

conditions expérimentales du diagnostic. Bien que la résolution et la précision de la mesure de densité soient moindres que celles obtenus dans le cas des mesures sur synchrotron, le diagnostic à l'aide d'un flash $\mathrm{X}$ constitue néanmoins une alternative attrayante dans le cas des applications visées.

\section{CARACTÉRISATION DES SPRAYS DE DODÉCANE}

La figure 2 présente les radiographies des premiers instants de la pénétration du spray de carburant dans la chambre d'injection à la pression atmosphérique. L'injecteur possède 6 orifices chacun d'un diamètre de $110 \mu \mathrm{m}$, la pression du rail est de 800 bars et un additif à base de cérium a été mélangé au dodécane. Des radiographies très similaires sont obtenues avec un autre injecteur dont les diamètres d'orifices sont de $150 \mu \mathrm{m}$, et les analyses faites à partir des clichés sont également semblables dans le cas de spray de dodécane pur [8]. Il a ainsi pour la première fois été effectué un diagnostic sur un carburant non dopé en élément lourd même si dans ce cas le contraste est plus faible et le rapport signal sur bruit détérioré. Cette expérience nous a servi de référence pour pouvoir valider l'analyse de jets enrichis en cérium dont l'ajout à quelques pour cent ne semble pas avoir une influence significative sur les propriétés d'atomisation en zone proche injecteur. A partir de la figure 2, nous avons déduit l'angle d'ouverture radiale du spray $\left(6^{\circ}\right)$ qui est constant en fonction de l'instant après le début de l'injection. L'analyse des profils de niveaux de gris suivant l'axe de propagation du jet, comme illustré sur la figure 3, révèle l'existence de deux zones de densités différentes. En sortie d'orifice, la densité décroit d'abord linéairement avec la distance, puis une zone de densité constante est mesurée jusqu'au front de pénétration du spray. Le profil effectué suivant un axe différent de celui d'expansion du spray, permet de vérifier l'homogénéité de l'illumination X de la CCD et d'estimer le rapport signal sur bruit des mesures.

Cette analyse effectuée pour des délais croissants par rapport au début de l'injection, permet de déduire une vitesse moyenne de propagation de la zone la plus dense de $45 \mathrm{~m} \cdot \mathrm{s}^{-1}$, tandis que le front se déplace à $130 \mathrm{~m} \cdot \mathrm{s}^{-1}$. Une accélération de la vitesse de chaque composante est mesurée au cours des premières centaines de $\mu$ s ce qui avait déjà été observé et attribué à l'établissement progressif du flux de carburant après la commande d'injection. Par ailleurs la mesure de la densité dans la zone proche de l'injecteur révèle que la fraction liquide est de $65 \%$ sur les premières centaines de micromètre en aval de l'orifice et décroit fortement avec la distance $(10 \%$ à $3 \mathrm{~mm})$. La fraction liquide désigne ici le rapport de la densité du spray par rapport à celle d'un jet de carburant à la densité du liquide. Ces résultats 


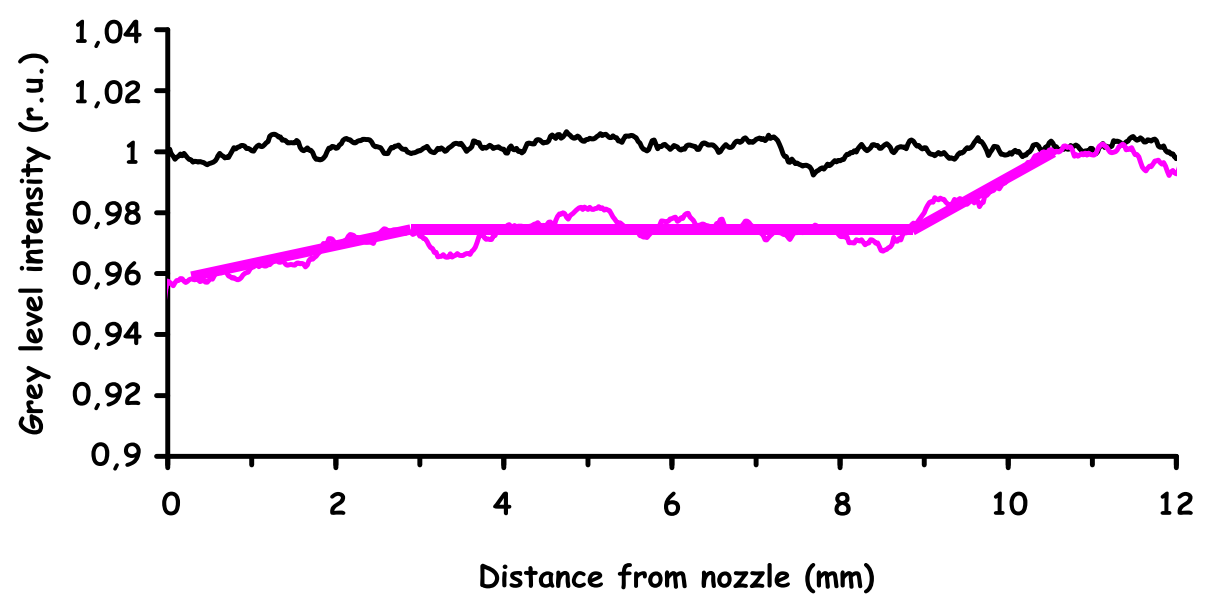

Figure 3. Profils de transmission longitudinale obtenus à partir de la radiographie de la figure 2 pour un délai de $80 \mu$ s. En noir hors de l'axe du spray, en clair et avec des traits pour la clarté du commentaire, suivant l'axe du jet.

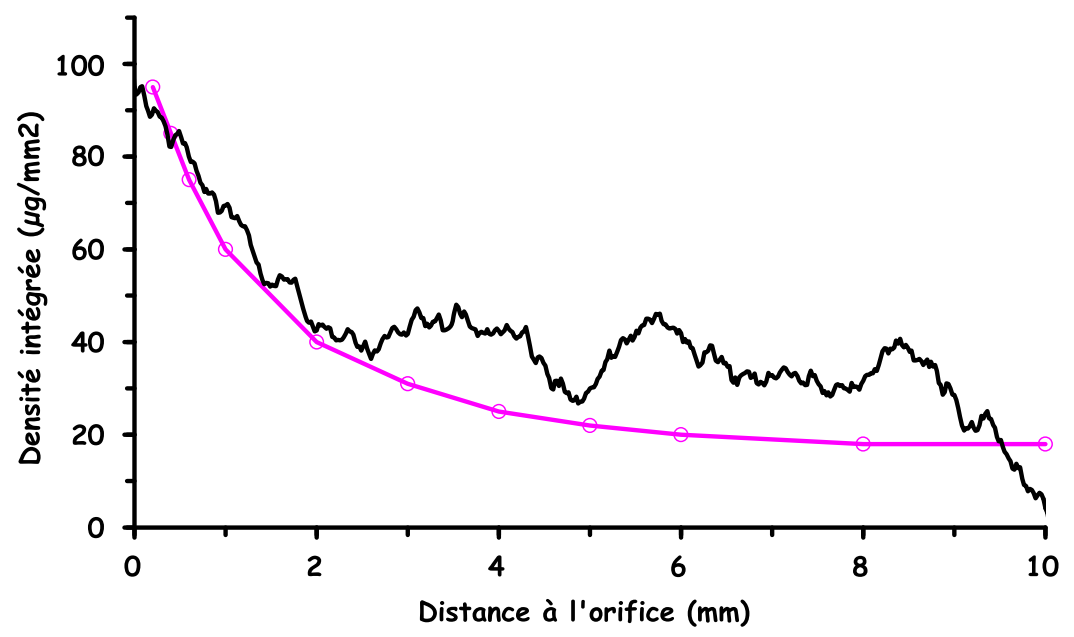

Figure 4. Evolution de la densité intégrée sur l'épaisseur du jet en fonction de la distance à l'orifice. En noir par flash X (injecteur 6 trous, $110 \mu \mathrm{m}$ ) en clair sur synchrotron [9] (injecteur 5 trous, $130 \mu \mathrm{m}$ ).

également en accord avec la bibliographie, témoignent d'un mélange assez efficace avec le gaz de la chambre d'injection dès les premiers millimètres d'expansion. Il apparaît ainsi que certains modèles qui considèrent un jet cylindrique purement liquide à la sortie des orifices avant que l'atomisation ne disperse le jet en microgouttelettes doivent être modifiés au regard des mesures par rayons X.

Le diagnostic par flash $\mathrm{X}$ permet ainsi l'étude dynamique des propriétés de sprays de diesel et l'essentiel des observations, qui soulignent tout l'intérêt d'un diagnostic par rayons X, confirment des mesures obtenus sur synchrotron. La figure 4 présente une comparaison de l'évolution de la densité intégrée sur l'épaisseur de jet en fonction de la distance à l'orifice obtenue par flash X et sur synchrotron pour un injecteur et des conditions d'injection proches. Bien que nos mesures soient moins précises, on note la très bonne concordance avec la technique de diagnostic $\mathrm{X}$ de référence tant d'un point de vue qualitatif que quantitatif. 


\section{CONCLUSIONS}

Le développement et l'utilisation d'une source compacte de type flash $\mathrm{X}$ a permis la radiographie et la caractérisation de sprays issus d'injecteur diesel de série. Plusieurs critères d'importance ont guidé l'optimisation du flash X pour l'adapter au mieux aux expériences de diagnostic de sprays d'un diamètre typique de $100 \mu \mathrm{m}$. Le domaine d'énergie favorable à l'obtention d'un contraste radiographique faible, quelques pour cent, mais optimal s'étend d'environ 7 à $10 \mathrm{keV}$. Ceci a conduit à optimiser une source $\mathrm{X}$ émettant un flux de photons important, émis sous forme d'impulsions nanosecondes, dans les raies $\mathrm{K}$ du cuivre à $8 \mathrm{keV}$. La caractérisation du jet micrométrique et de faible contraste aux rayons $\mathrm{X}$, a été menée à l'aide d'une source $\mathrm{X}$ dont le foyer émetteur d'un diamètre de quelques centaines de $\mu \mathrm{m}$ est situé à $75 \mathrm{~cm}$ de l'axe du spray. L'image radiographique, enregistrée sur un CCD X, est le résultat d'une moyenne sur un à plusieurs milliers d'injections synchronisées avec le flash $\mathrm{X}$. Une résolution spatiale de $120 \mu \mathrm{m}$ a été mesurée dans les conditions expérimentales. Le spectre $\mathrm{X}$ émis principalement dans les raies caractéristiques comporte néanmoins une composante continue de freinage. La mesure de l'énergie moyenne équivalente du spectre du flash X, à l'aide d'objets test de dimension et densité proches de celle des sprays, fournit une valeur de $8,3 \mathrm{keV}$. La validation et la mesure de la précision du diagnostic de densité d'objets micrométrique à l'aide du flash $\mathrm{X}$, en intégrant cette valeur moyenne équivalente de l'énergie, ont été réalisées sur des fils de nylon. Dans les conditions expérimentales retenues, l'écart dans la détermination de la densité est inférieur à $15 \%$ pour des objets d'une dimension supérieure à $100 \mu \mathrm{m}$. Au cours de ces travaux, nous avons réalisé les premières radiographies sur des sprays de dodécane pur, sans dopant [8]. A partir de ces radiographies, un angle d'ouverture radial du jet de $5^{\circ}$, une vitesse d'expansion de la zone la plus dense d'environ $40 \mathrm{~m} . \mathrm{s}^{-1}$ et du front avant du spray d'environ $150 \mathrm{~m} . \mathrm{s}^{-1}$, ainsi qu'une fraction liquide de $60 \%$ ont été mesuré. Nos études montrent aussi que l'ajout de cérium, élément de numéro atomique élevé, permet d'augmenter significativement le contraste des sprays sans perturber les propriétés d'écoulements ce qui permet d'envisager de nouvelles caractérisation sur des jets plus fortement dopés, pour lesquels le rapport signal sur bruit des radiographies pourrait être largement amélioré. La comparaison de nos résultats avec ceux obtenus récemment dans des conditions d'injection réalistes et proches des nôtres sur les anneaux synchrotron révèle des interprétations et mesures en très bon accord. Les propriétés de l'écoulement, angle d'ouverture de la zone dense et vitesses de propagation ainsi que la valeur et l'évolution de la densité du jet en fonction de la distance à l'orifice d'injection sont ainsi obtenus à partir d'une source $\mathrm{X}$ compacte et facilement accessible. Des expériences de caractérisation des sprays diesel ont été effectuées avec succès jusqu'à des pressions de remplissage de l'enceinte à haute pression de 30 bars ce qui permet d'approcher les conditions de fonctionnement moteur. Le flux de photons $\mathrm{X}$ atteignant le $\mathrm{CCD}$ est bien sûr beaucoup plus faible, le rapport signal sur bruit détérioré mais les radiographies permettent néanmoins la visualisation et la caractérisation du spray. La possibilité d'obtenir des mesures expérimentales dans des conditions de très faible contraste, devrait aussi offrir l'opportunité d'effectuer un diagnostic conjoint, avec des zones de recouvrement, exploitant à la fois la radiographie $\mathrm{X}$ de basse énergie et les techniques optiques plus conventionnelles. L'utilisation d'une source $\mathrm{X}$ couplée à une optique de focalisation ou de mise en forme d'un faisceau $\mathrm{X}$ à faible divergence serait également une extension envisageable à nos études pour augmenter la résolution spatiale du diagnostic.

\section{Remerciements}

Les auteurs remercient G. Blokkeel (PSA) et F.X. Demoulin (CORIA) pour leurs suggestions et leur support dans l'analyse des résultats obtenus par le diagnostic par rayons $\mathrm{X}$ au cours du programme Emphase. 


\section{Références}

[1] C. F. Powell, Y. Yue, R. Poola, and J. Wang, "Time-resolved measurements of supersonic fuel sprays using synchrotron X-rays”, J. Synchrotron Rad., vol. 7, pp. 356-360, 2000.

[2] Y. Y. Yue, C. F. Powell, R. Poola, and J. Wang, J. K. Schaller, "Quantitative measurements of diesel fuel spray characteristics in the near-nozzle region using x-ray absorption", Atomization and sprays, vol. 11, pp. 471-490, 2001.

[3] J. Wang, "X-ray vision of fuel sprays", ”, J. Synchrotron Rad., vol. 12, pp. 197-207, 2005.

[4] A. L. Kastengren, C. F. Powell, Y. Wang, K.-S. Im, and J. Wang, "X-ray radiography measurements of diesel spray structure at engine-like ambient density" ILASS Americas, $21^{\text {st }}$ annual conference on liquid atomization and spray systems, Orlando, FL, May 2008.

[5] J. M. Pouvesle, C. Cachoncinlle, R. Viladrosa, E. Robert and A. Khacef, "Compact flash X-ray sources and their applications", Nucl. Instrum. Meth. Phys. B, vol. 113, 134, 1996.

[6] E. Romero, C. Cachoncinlle, E. Robert, R. Viladrosa, S. Dozias, G. Coudrat, and J.M. Pouvesle, "Characterization and optimization of a flash X-ray source for diagnostic of dense sprays", UVX2008, EDP Sciences, DOI:10.1051/uvx/2009029, pp. 177-181, 2009.

[7] J. J. Curry, "Quantitative X-ray absorption imaging with a broadband source: application to highintensity discharge lamps”, J. Phys. D: Appl. Phys., vol. 41, 144020, 2008.

[8] E. Robert, S. Dozias, R. Viladrosa, C. Cachoncinlle and J.M. Pouvesle, "Table-top flash X-ray diagnostics of dodecane sprays", ILASS-Europe 2010, $23^{\text {rd }}$ Annual Conference on Liquid Atomization and Spray Systems, Brno, Czech Republic, 2010.

[9] P. Leick, A. L. Kastengren, Z. Liu, J. Wang, and C. F. Powell, "X-ray measurements of mass distribution in the near-nozzle region of sprays from standard multi-hole common-rail diesel injection systems", ICLASS 2009, $11^{\text {th }}$ triennial International Annual Conference on Liquid Atomization and Spray Systems, Vail, Colorado, 2009. 